\title{
Implementation of a nursing home care management pathway developed to facilitate the use of the Malnutrition Universal Screening Tool
}

\author{
A. Molyneux ${ }^{1}$, H. $\operatorname{Hogan}^{2}$ and A. Burns ${ }^{1}$ \\ ${ }^{1}$ Care Home Services, Glasgow G41 1AF, UK and ${ }^{2}$ Prescribing Support Unit, Glasgow G42 9TT, UK
}

The following work was undertaken to provide an evidence base for the introduction and implementation of a recently developed, Care Homes Management Clinical Care Pathway based around Step 5 of The Malnutrition Universal Screening Tool (MUST). The main aim is to aid practitioners to manage residents' nutrition who have been identified at moderate to high risk of undernutrition. The ability of staff to provide dietary advice in an appropriate manner is the most important factor in patient satisfaction ${ }^{(1)}$. It is envisaged that the guidance tool will support practitioners within the multidisciplinary team to be effective in providing a targeted approach to managing undernutrition using food and fluid and the appropriate use of nutritional supplements.

A baseline clinical audit and second audit cycle 6 months later was undertaken in the same group of nursing care homes.10 homes were invited to participate, a random sample of 10 care plans and associated documentation were assessed against national clinical care standards, using an audit tool modified for long-term care settings ${ }^{(2)}$.

Data analysis was undertaken by the Clinical Governance Support Unit for the baseline audit in July 2009 and subsequently for the second audit cycle in December 2009. Education sessions to support the use of MUST and the developed clinical care pathway were delivered and a nutrition champion to lead the implementation within their nursing home was identified.

The key elements assessed included: resident dietary assessment, nutritional screening and resident care planning.

\begin{tabular}{|c|c|c|c|}
\hline Key elements & Baseline audit & 2nd audit & Difference \\
\hline $\begin{array}{l}\text { - Were factors that affect food intake recorded at initial assessment e.g., environmental? } \\
\text { - Was the resident assessed using the MUST tool? } \\
\text { - Was their evidence of food fortification for } 4 \text { weeks for moderate to high-risk residents? }\end{array}$ & $\begin{array}{l}39 \%\left(39^{/ 100}\right) \\
68 \%\left(68^{/ 100}\right) \\
20 \%\left(5^{/ 25}\right) \mathrm{MR}^{*} \\
8 \%\left(2^{/ 24}\right) \mathrm{HR}^{*}\end{array}$ & $\begin{array}{l}78 \%\left(78^{1100}\right) \\
99 \%\left(99^{100}\right) \\
67 \%\left(8^{/ 12}\right) \mathrm{MR} \\
63 \%\left(17^{227}\right) \mathrm{HR}\end{array}$ & $\begin{array}{l}\text { Increase of } 39 \% \\
\text { Increase of } 31 \% \\
\text { Increase of } 47 \% \mathrm{MR}, 55 \% \mathrm{HR}\end{array}$ \\
\hline
\end{tabular}

*MR, moderate risk; HR, high risk.

There was an overall improvement in documentation and care planning in all areas.

When compared with the baseline figures the 2nd audit cycle showed an improvement in the residents' nutritional risk scores within the population group as follows: low risk $26-61 \%$, moderate risk $38-12 \%$ and high risk $35-27 \%$.

These results indicate that using a systematic, multidisciplinary approach alongside education and tools to facilitate practice can reduce the nutrition risk status of care home residents. This would confer with previous authors ${ }^{(1,3)}$.

Early identification of moderate- to high-nutritional risk residents' using MUST and the use of a clear care pathway using food first advice as a first line measure, along with referral guidance can benefit the nutritional outcomes of the resident. This has been demonstrated with the use of this MUST nursing home care pathway and guidance.

1. National Institute for Clinical Excellence, NICE (2006) Nutritional Support for Adults, Oral Nutritional Support, Enteral Tube Feeding and Parental Nutrition. Clinical Guideline 32

2. Quality Improvement Scotland: Food, Fluid and Nutritional Care in Scotland, September (2003).

3. Health and Community Care: Older People Living in the Community - Nutritional Needs, Barriers and Interventions: a literature review (2009). 